\title{
$d$-Wave Superconductivity in a System with Open Boundary Conditions
}

\author{
P. PisArski and G. Harań \\ Institute of Physics, Politechnika Wrocławska \\ Wyb. Wyspiańskiego 27, 50-370 Wrocław, Poland
}

\begin{abstract}
We discuss the $d$-wave superconductivity in confined geometry by imposing open boundary conditions on the attractive Hubbard model. Within the Bogoliubov-de Gennes approach we evaluate the order parameter and charge density distribution in the system.
\end{abstract}

PACS numbers: 74.20.Rp, 74.72.-h, 74.78.Na, 74.81.--g

\section{Introduction}

Constant progress in designing superconducting nanoscale devices, like built on superconducting junctions quantum bits [1], stimulates studies of superconductivity in confined geometries. The interest in this issue is also spurred by related problems of superconductivity at the edge of a bulk sample or surface superconductivity possibly relevant in tunneling experiments in cuprates [2]. Physics of superconductivity in confined geometry has been discussed for the $s$-wave system approximated by a square lattice with open boundary conditions [3, 4]. Here we consider the development of the $d$-wave superconductivity on such a model lattice.

\section{Hamiltonian and boundary conditions}

We define the system by the attractive Hubbard Hamiltonian which supports the $d$-wave superconductivity [5-7]:

$$
\begin{aligned}
H= & -t \sum_{\langle i, j\rangle \sigma}\left(c_{i \sigma}^{\dagger} c_{j \sigma}+\text { h.c. }\right)-\mu \sum_{i \sigma} n_{i \sigma} \\
& +U_{0} \sum_{i} n_{i \uparrow} n_{i \downarrow}+\frac{U_{1}}{2} \sum_{\langle i, j\rangle \sigma \sigma^{\prime}} n_{i \sigma} n_{j \sigma^{\prime}},
\end{aligned}
$$

where $c_{i \sigma}^{\dagger}\left(c_{i \sigma}\right)$ is the fermionic creation (annihilation) operator with spin $\sigma$ on a site $r_{i}$ of a square lattice, $t$ is the nearest-neighbor hopping, $U_{0}>0$ is the on-site 
repulsion potential, $U_{1}<0$ is the pairing interaction, $n_{i \sigma}=c_{i \sigma}^{\dagger} c_{i \sigma}$, and $\mu$ is the chemical potential. Symbol $\langle i j\rangle$ means the nearest-neighbor summation.

We proceed by performing the mean-field approximation [5-7] which leads to an effective quadratic Hamiltonian

$$
\begin{aligned}
H_{\mathrm{eff}} & =-\sum_{i \delta \sigma}\left(\frac{1}{2} \tilde{t}_{i i+\delta} c_{i \sigma}^{\dagger} c_{i+\delta \sigma}+\text { h.c. }\right)-\sum_{i \sigma} \tilde{\mu}_{i \sigma} n_{i \sigma}+\sum_{i}\left(\Delta\left(r_{i}\right) c_{i \uparrow}^{\dagger} c_{i \downarrow}^{\dagger}+\text { h.c. }\right) \\
& +\sum_{i \delta}\left(\frac{1}{2} \Delta\left(r_{i+\delta i}\right) c_{i+\delta \uparrow}^{\dagger} c_{i \downarrow}^{\dagger}+\frac{1}{2} \Delta\left(r_{i i+\delta}\right) c_{i \uparrow}^{\dagger} c_{i+\delta \downarrow}^{\dagger}+\text { h.c. }\right)
\end{aligned}
$$

where $\tilde{t}_{i i+\delta}=t+\frac{U_{1}}{4} \sum_{\sigma}\left\langle c_{i+\delta \sigma}^{\dagger} c_{i \sigma}\right\rangle, \tilde{\mu}_{i \uparrow(\downarrow)}=\mu-U_{0}\left\langle n_{i \downarrow(\uparrow)}\right\rangle-\frac{U_{1}}{2} \sum_{\delta \sigma}\left\langle n_{i+\delta \sigma}\right\rangle$, $\Delta\left(r_{i}\right)=U_{0}\left\langle c_{i \downarrow} c_{i \uparrow}\right\rangle, \Delta\left(r_{i i+\delta}\right)=U_{1}\left\langle c_{i+\delta \downarrow} c_{i \uparrow}\right\rangle$, and the summation index $\delta$ embraces the $i$-site nearest-neighbors. The effective Hamiltonian is diagonalized by the Bogoliubov-de Gennes (BdG) transformation [8] and solved on lattices of sizes up to $32 \times 32$ with open boundary conditions (OBC) imposed.

We define the open boundary conditions by the constraints $\tilde{t}_{i j}=0$ and $\Delta\left(r_{i j}\right)=0$ for $i$ or $j$ beyond the square lattice, i.e., we bind the order parameter and the hopping integral to vanish at the edge of the system.

Before proceeding, we comment on the choice of used parameters. We have studied the system for a range of parameters $0.5 \leq\left|U_{1} / t\right| \leq 1.0$ (weak pairing) and $1.0<\left|U_{1} / t\right| \leq 3.0$ (strong pairing) taking $U_{0}=-U_{1}$. The average charge density has been set near and at half-filling to picture the high-temperature superconductors. For such a filling we also expect the effect of the Hartree potential to be the least significant, which is important as our model Hamiltonian does not comprehend the spin dynamics and cannot properly take the Hartree shifts into account. In the following the Hartree corrections are neglected in our numerical calculations.

\section{Order parameter and particle density}

Breaking of the tetragonal symmetry by OBC can be perceived as a formation of the $s$-wave component of the $d$-wave order parameter, a component which is invariant under a whole group of tetragonal transformations. Given the order parameter around the $r_{i}$ site $(\delta=x, y,-x,-y)$ of the lattice $\Delta_{\delta}\left(r_{i}\right)=\left[\Delta\left(r_{i i+\delta}\right)+\Delta\left(r_{i+\delta i}\right)\right] / 2$, we identify the $s$-wave component as $\Delta_{s}\left(r_{i}\right)=\left[\Delta_{x}\left(r_{i}\right)+\Delta_{-x}\left(r_{i}\right)+\Delta_{y}\left(r_{i}\right)+\Delta_{-y}\left(r_{i}\right)\right] / 4$, whereas the proper $d$-wave component is defined as $\Delta_{d}\left(r_{i}\right)=\left[\Delta_{x}\left(r_{i}\right)+\Delta_{-x}\left(r_{i}\right)-\Delta_{y}\left(r_{i}\right)-\Delta_{-y}\left(r_{i}\right)\right] / 4$. These two counterparts of the order parameter are evaluated for a $22 \times 22$ square lattice with OBC by solving the BdG equations [8]. We show the $d$-wave component $\Delta_{d}\left(r_{i}\right)$ and compare it to the solution of the system with periodic boundary conditions $(\mathrm{PBC})$ at half-filling $(n=1.0)$ for the weak-pairing in Figs. 1a,b, and for the strong-pairing in Figs. 1c,d. The corresponding $s$-wave solutions are shown in Fig. 2. Let us note the wide-spread oscillations of the order parameter for the 
weak-pairing superconductivity. The charge density distribution for the average particle density $n=0.9$ is presented in Fig. 3 , for the half-filling case the particle density function is constant $n\left(r_{i}\right)=1$.
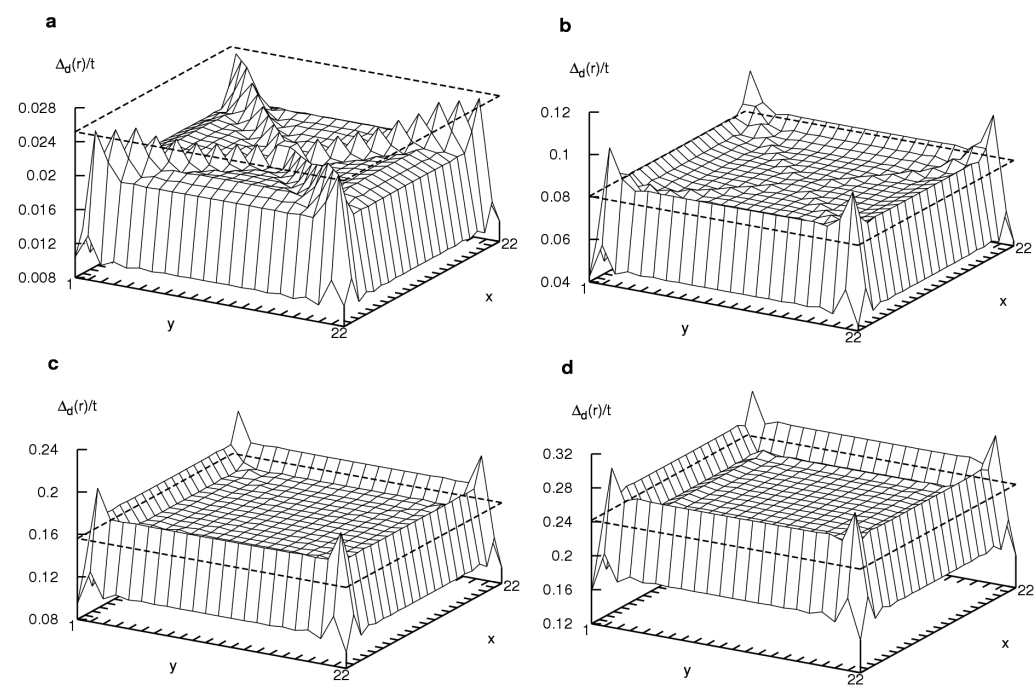

Fig. 1. The $d$-wave component $\Delta_{d}\left(r_{i}\right)$ of the order parameter on a square lattice $22 \times 22$ with $\mathrm{OBC}$ (solid lines) and PBC (dashed lines) for the average charge density $n=1.0$, the on-site repulsion potential $U_{0}$, the $d$-wave pairing potential $U_{1}$ : (a) $U_{0}=-U_{1}=0.5 t$, (b) $U_{0}=-U_{1}=1.0 t$, (c) $U_{0}=-U_{1}=1.5 t$, (d) $U_{0}=-U_{1}=2.0 t$.
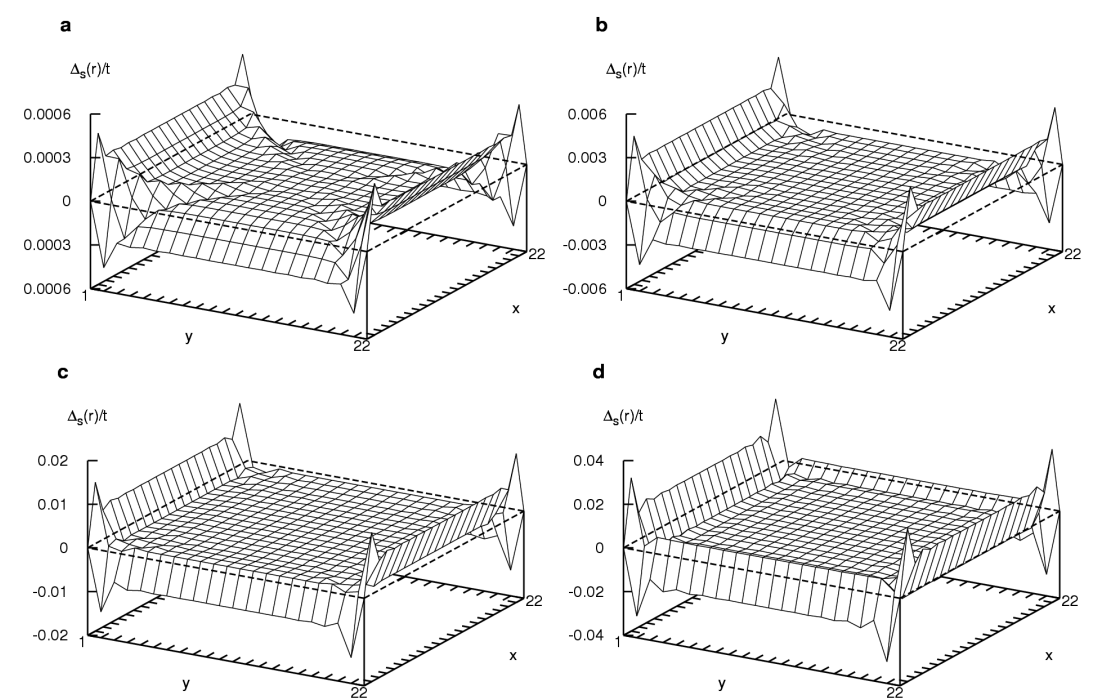

Fig. 2. The $s$-wave component $\Delta_{s}\left(r_{i}\right)$ of the order parameter for OBC (solid lines) and PBC (dashed lines) on the lattice as in Fig. 1. 

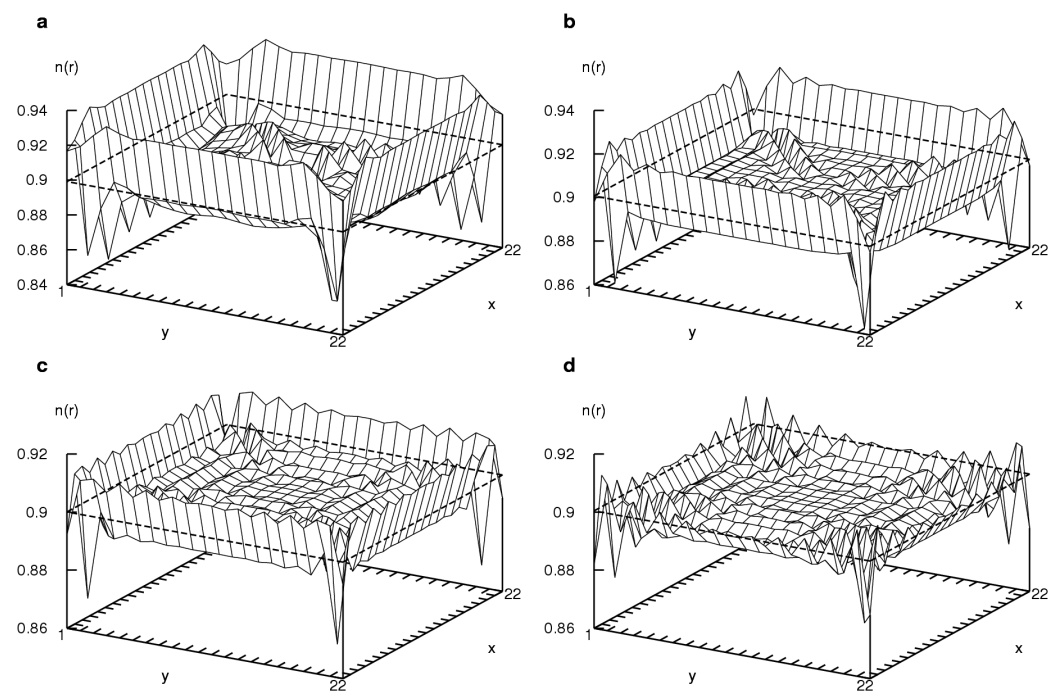

Fig. 3. The charge density $n\left(r_{i}\right)$ distribution in the $d$-wave superconducting system on a square lattice $22 \times 22$ with OBC (solid lines) and PBC (dashed lines) for the average charge density $n=0.9$ and the potential parameters as in Fig. 1.

\section{Conclusions}

We showed that the open boundary conditions, similarly to the impurity [7], break the rotational and translational invariance of the system leading to a formation of the $s$-wave component of the $d$-wave order parameter. Our calculations report a significant variation of $\Delta_{d}\left(r_{i}\right)$ and $\Delta_{s}\left(r_{i}\right)$ order parameters, and the particle density function $n\left(r_{i}\right)$ near the edge of a sample. Although developed locally on the edge of the lattice, these Friedel-like oscillations extend towards the center of the sample for weak pairing.

\section{Acknowledgments}

This work was supported in part by the grant No. PBZ-MIN-008/PO3/03.

\section{References}

[1] Y. Makhlin, G. Schön, A. Shnirman, Rev. Mod. Phys. 73, 357 (2001).

[2] K. McElroy, R.W. Simmonds, J.E. Hoffman, D.-H. Lee, J. Orenstein, H. Eisaki, S. Uchida, J.C. Davis, Nature 422, 592 (2003).

[3] K. Tanaka, F. Marsiglio, Physica C 384, 356 (2003).

[4] K. Tanaka, F. Marsiglio, J. Phys. Chem. Solids 63, 2287 (2002). 
[5] J.X. Zhu, B. Friedman, C.S. Ting, Phys. Rev. B 59, 3353 (1999).

[6] A. Ghosal, M. Randeria, N. Trivedi, Phys. Rev. B 65, 014501 (2001).

[7] M. Franz, C. Kallin, A.J. Berlinsky, Phys. Rev. B 54, 6897 (1996).

[8] P.G. de Gennes, Superconductivity of Metals and Alloys, Addison-Wesley Publ. Co., Redwood City, CA 1989. 